\title{
Stupéfiants, circulation routière et LC-MS/MS
}

\section{Illégal drugs, road traffic and LC-MS/MS}

Annie CAILLEUX*, Anne LE BOUIL, Alain TURCANT, Bertrand DIQUET

Service de Pharmacologie et Toxicologie, Centre Hospitalier Universitaire, 4, rue Larrey 49933 ANGERS Cedex 09 - FRANCE

* Auteur à qui adresser la correspondance : Annie CAILLEUX, Service de Pharmacologie et Toxicologie, Centre Hospitalier Universitaire, 4, rue Larrey - 49933 ANGERS Cedex 09 - FRANCE Tél : 0241354553 - Fax : 0241354877 - E-mail : ancailleux@chu-angers.fr

(Reçu le 15 janvier 2005 ; accepté après modifications le 18 janvier 2005)

\section{$R E$ SUME}

En France, depuis quelques années, des recherches de stupéfiants chez les conducteurs impliqués dans un accident ont été mises en cuvre dans le cadre de la sécurité routière. Ces composés : les cannabinoüdes, les amphétamines, les opiacés et la cocaüne et ses métabolites se trouvent dans le sang à de faibles concentrations pour lesquelles il est nécessaire de mettre en œuvre des techniques analytiques très spécifiques et sensibles. Les stupéfiants sont la plupart du temps analysés par chromatographie en phase gazeuse couplée à la spectrométrie de masse après extraction liquidelliquide ou extraction sur phase solide suivie par une étape de dérivation (silylation ou alkylation). Nous avons choisi de mettre en place la recherche des cannabinoüdes, des opiacés et de la cocaïne et de ses métabolites par spectrométrie de masse en tandem couplée à la chromatographie liquide (LC$M S / M S)$. Cette méthode comporte une étape d'ionisation à pression atmosphérique qui peut se faire soit en phase liquide par source electrospray (ESI) soit en phase vapeur par ionisation chimique en utilisant un nébuliseur chauffé $(A P C D)$ ou une source photospray (APPI) en fonction de la polarité des molécules. Les cannabinoïdes ont été ionisés en utilisant le mode APCI ou le mode APPI; les opiacés, la cocaïne et ses métabolites ont été ionisés par source electrospray. La méthode a été validée pour des concentrations comprises entre $0,5 \mu \mathrm{g} / \mathrm{l}$ et $100 \mu \mathrm{g} / \mathrm{l}$ pour les cannabinoïdes et entre $10 \mu \mathrm{g} / \mathrm{l}$ et $1000 \mu \mathrm{g} / \mathrm{l}$ pour les autres stupéfiants.

\section{MOTS-CLÉS}

Stupéfiants, cannabinoïdes, opiacés, cocaïne, benzoylecgonine, méthylecgonine, LC-MS/MS, APCI, APPI, ESI.

\section{SUMMARY}

Recently, French laws have introduced obligation of measuring drugs of abuse levels on drivers involved in car accident. High sensitivity of analytical technique is required because blood concentrations of these compounds: cannabinoids, amphetamines, opiates, cocaine and its metabolites are low and decrease rapidly. Their determinations are usually performed by gas chromatography'/mass spectrometry (GC/MS) after liquid/liquid or solid phase extraction and derivatisation (silylation or alkylation). In this study we propose a method using liquid chromatography connected to tandem mass spectrometry (LC-MS/MS) to detect cannabinoids, opiates, cocaine and its metabolites in blood of drivers. This method needs an atmospheric pressure ionization source depending of the polarity of molecules and using electrospray source (ESI) for polar compounds, a heated nebulizer (APCI) or a photospray source (APPI) for less polar compounds. Cannabinoids were ionized using APCI or APPI mode, opiates, cocaine and metabolites were ionized using electrospray mode. Validation was done for concentrations between $0.5 \mu \mathrm{g} / \mathrm{l}$ and $100 \mu \mathrm{g} / \mathrm{l}$ for cannabinoids and between $10 \mu \mathrm{g} / \mathrm{l}$ and $1000 \mu \mathrm{g} / \mathrm{l}$ for other illegal drugs.

\section{KEY-WORDS}

Illegal drugs, cannabinoids, opiates, cocaine, benzoylecgonine, methylecgonine, LC-MS/MS, APCI, APPI, ESI. 


\section{Introduction}

En 1999, les autorités judiciaires ont instauré un dépistage systématique de stupéfiants sur tout conducteur impliqué dans un accident mortel de la circulation routière (1). Par la suite, le dépistage a été étendu aux conducteurs impliqués dans un accident corporel. Au début de 2003, a été mis en place un délit de conduite après usage de stupéfiants, entraînant des sanctions (2). Les :recherches mises en œuvre dans le cadre de la sécurité routière concernent 4 classes de substances ou plantes classées comme stupéfiants : les cannabinoïdes, les amphétamines, les opiacés et la cocaïne et ses métabolites. Ces composés sont la plupart du temps analysés par chromatographie en phase gazeuse couplée à la spectrométrie de masse (3 -12).

La spectrométrie de masse en tandem couplée à la chromatographie liquide (LC-MS/MS) est une méthode sensible et extrêmement spécifique et nous a semblé être une technique de choix pour effectuer ces analyses (13-28). Cette méthode comporte une étape d'ionisation à pression atmosphérique qui peut se faire soit en phase liquide par source electrospray (ESI) soit en phase vapeur par ionisation chimique en utilisant un nébuliseur chauffé (APCI) ou une source photospray (APPI). Selon la nature et la polarité des molécules analysées, il peut être intéressant d'utiliser l'un ou l'autre des modes d'ionisation. Les cannabinoïdes ont été ionisés en utilisant le nébuliseur chauffé, les autres stupéfiants ont été ionisés par source electrospray.

\section{Matériel et méthodes}

\section{Réactifs}

Les solvants (chloroforme, isopropanol, éther, hexane, méthanol) ainsi que l'ammoniaque, les acides formique, acétique et le formiate d'ammonium sont de qualité pour analyses et proviennent de Merck (Darmstadt, Allemagne).

L'acétonitrile de qualité pour chromatographie a été fourni par Prolabo (France).

Les opiacés : morphine, codéine, norcodéine, codéthyline, pholcodine, 6-monoacétylmorphine ainsi que la cocaïne, la benzoylecgonine et la méthylecgonine, sont des produits Sigma (St Quentin Fallavier, France).

Les cannabinoïdes : tétrahydrocannabinol (THC), hydroxytétrahydrocannabinol (11-OH THC), acide tétrahydrocannabinoïque (THC-COOH) ainsi que tous les analogues deutérés : morphine, codéine, benzoylecgonine, méthylecgonine, térahydrocannabinol, acide tétrahydrocannabinoïque ont été obtenus en solution à $100 \mu \mathrm{g} / \mathrm{ml}$ chez Promochem.

\section{Matériel}

La séparation chromatographique est réalisée à l'aide d'un chromatographe en phase liquide série 200 LC Perkin-Elmer (Les Ulis, France) constitué de deux micro pompes, d'un compartiment thermostaté, d'un injecteur automatique et de colonnes chromatographiques $\mathrm{C} 8(5 \mu \mathrm{m}, 100 \times 2,1 \mathrm{~mm}$ d.i.). La phase mobile est constituée d'un mélange acétonitrile et eau contenant $2 \mathrm{mM}$ de formiate d'ammonium et $25 \mathrm{mM}$ d'acide formique.

Le spectromètre de masse est un triple quadripôle API 300 Sciex Applied Biosystem (Les Ulis, France) équipé de deux sources d'ionisation à pression atmosphérique : 1'electrospray (ESI) et le nébuliseur chauffé pour l'ionisation chimique (APCI).

L'ionisation est faite en mode positif pour tous les stupéfiants. Les tensions d'ionisation, d'orifice ainsi que les énergies de collisions ont été optimisées pour chaque molécule.

Le mode d'acquisition des données est le MRM (multiple reaction monitoring) qui consiste à analyser chaque composé à l'aide d'un couple spécifique d'ions constitué d'un ion moléculaire protoné et d'un fragment issu de cet ion. Les valeurs correspondant à ces transitions sont reportées sur le tableau I pour les cannabinoïdes et sur le tableau II pour les opiacés et la cocaïne et ses métabolites.

Tableau I : Transitions MRM pour les cannábinoüdes.

\begin{tabular}{|c|c|c|}
\hline composé & $\begin{array}{c}\text { Ion molécuaire protoné } \\
(\mathbf{m} / \mathbf{z})\end{array}$ & $\begin{array}{c}\text { Fragment } \\
(\mathbf{m} / \mathbf{z})\end{array}$ \\
\hline THC & 315.1 & 193.0 \\
OH-THC & 331.1 & 313.0 \\
THC-COOH & 345.1 & 299.2 \\
\hline
\end{tabular}

Tableau II : Transitions MRM pour opiacés, cocaïne et ses métabolites.

\begin{tabular}{|c|c|c|}
\hline composé & $\begin{array}{c}\text { Ion moléculaire protoné } \\
(\mathbf{m} / \mathbf{x})\end{array}$ & $\begin{array}{c}\text { Fragment } \\
(\mathbf{m} / \mathbf{z})\end{array}$ \\
\hline Morphine & 286.2 & 165.1 \\
Norcodéine & 286.2 & 165.1 \\
6-MAM & 328.2 & 165.1 \\
Codéine & 300.2 & 165.1 \\
Codéthyline & 314.2 & 165.1 \\
Pholcodine & 399.3 & 114.1 \\
Cocaïne & 304.2 & 182.2 \\
Benzoylecgonine & 290.2 & 168.2 \\
Méthylecgonine & 200.2 & 182.2 \\
\hline
\end{tabular}




\section{Préparation des échantillons}

Toutes les analyses sont faites sur du sang total conservé au congélateur à $-20^{\circ} \mathrm{C}$.

\section{Cannabinoüdes}

Dans un tube de verre, sur $1 \mathrm{ml}$. de sang sont ajoutés $20 \mu l$ d'une solution d'étalons internes (THC-D3, THC-COOH-D3) à $1 \mathrm{mg} / \mathrm{l}, 200 \mu \mathrm{l}$ d'acide acétique à $10 \%$ et $3,5 \mathrm{ml}$ d'un mélange d'hexane et d'acétate d'éthyle (80/20). Après 15 min d'agitation sur agitateur rotatif, la phase organique est prélevée, évaporée à sec et reprise par $100 \mu \mathrm{l}$ de méthanol. $20 \mu \mathrm{l}$ sont injectés sur la colonne.

\section{Opiacés, cocaüne et métabolites}

Dans un micro tube à usage unique de $2 \mathrm{ml}$ sont ajoutés $250 \mu \mathrm{l}$ de sang, $25 \mu \mathrm{l}$ d'une solution d'étalons internes (morphine-D3, codéine-D3, benzoylecgonineD3, méthylecgonine-D3) à $2,5 \mathrm{mg} / \mathrm{l}, 100 \mu \mathrm{l}$ de tampon ammoniaque $(1 \mathrm{M}, \mathrm{pH}$ 9) et $1,25 \mathrm{ml}$ de phase organique constituée d'un mélange de chloroforme et d'isopropanol $(95 / 5, \mathrm{~V} / \mathrm{V})$. Après 3 min d'agitation puis centrifugation, la phase organique est récupérée et évaporée à sec. Le résidu est repris par $100 \mu \mathrm{l}$ d'un mélange d'eau et d'acétonitrile (5/1, V/V). $20 \mu \mathrm{l}$ sont injectés dans le système chromatographique.

\section{Validation}

La linéarité a été étudiée à 7 niveaux de concentration pour les opiacés, la cocaïne et ses métabolites $(10$; $25 ; 50 ; 100 ; 250 ; 500 ; 1000 \mu \mathrm{g} / \mathrm{l})$ et à des concentrations plus faibles pour les cannabinoïdes 0,$5 ; 1$; $5 ; 10 ; 25 ; 50 ; 100 \mu \mathrm{g} / \mathrm{l})$.

La fidélité a été testée en surchargeant du sang total à 3 niveaux de concentration $(25 ; 100 ; 1000 \mu \mathrm{g} / \mathrm{l})$ pour les opiacés, la cocaïne et ses métabolites. Pour les cannabinoïdes, 4 niveaux de concentration $(0,5 ; 1 ; 10$; $100 \mu \mathrm{g} / \mathrm{l})$ ont été étudiés pour le THC et 3 niveaux de concentration ( 1 ou $5 ; 10 ; 100 \mu \mathrm{g} / \mathrm{l})$ pour le THC$\mathrm{COOH}$ et le $\mathrm{OH}-\mathrm{THC}$ respectivement. La répétabilité a été évaluée en analysant 6 fois chaque échantillon le jour de sa préparation. La reproductibilité a été évaluée en analysant chaque échantillon 6 jours différents pour les opiacés, la cocaïne et ses métabolites et seulement 5 jours différents pour les cannabinoïdes.

La justesse a été évaluée par la méthode des pourcentages de récupération sur 6 sangs différents avant et après surcharges aux concentrations testées pour la fidélité.

\section{Résultats et discussion}

\section{Les opiacés, la cocaïne et ses métabolites}

Ces composés ont été ionisés par méthode electrospray.

La morphine et la norcodéine sont des isomères et se fragmentent de la même façon. Il est donc nécessaire d'obtenir leur séparation chromatographique. Un exemple de séparation est présenté sur la figure 1 sur un échantillon surchargé à $10 \mu \mathrm{g} / \mathrm{l}$.

La cocaïne et ses métabolites sont des composés qui se fragmentent facilement et permettent d'obtenir un bon rapport signal/bruit pour de faibles concentrations. Un exemple d'échantillon surchargé à $10 \mu \mathrm{g} / \mathrm{l}$ par la benzoylecgonine est présenté sur la figure 2 .

Les résultats de validation ont déjà été décrits pour les opiacés, la cocaïne et ses métabolites $(14,15)$.

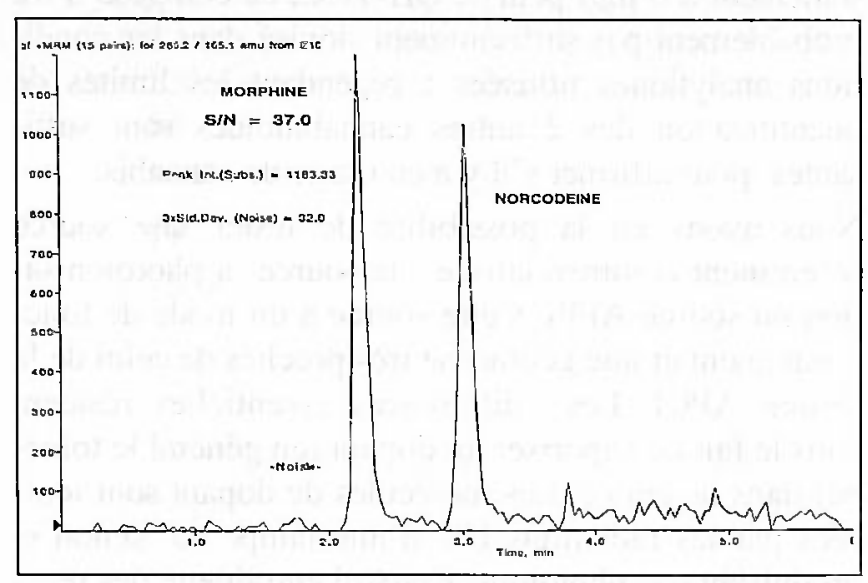

Figure 1 : Chromatogranme d'un extrait sanguin surchargé par $10 \mu \mathrm{g} / \mathrm{l}$ de morphine et norcodéine.

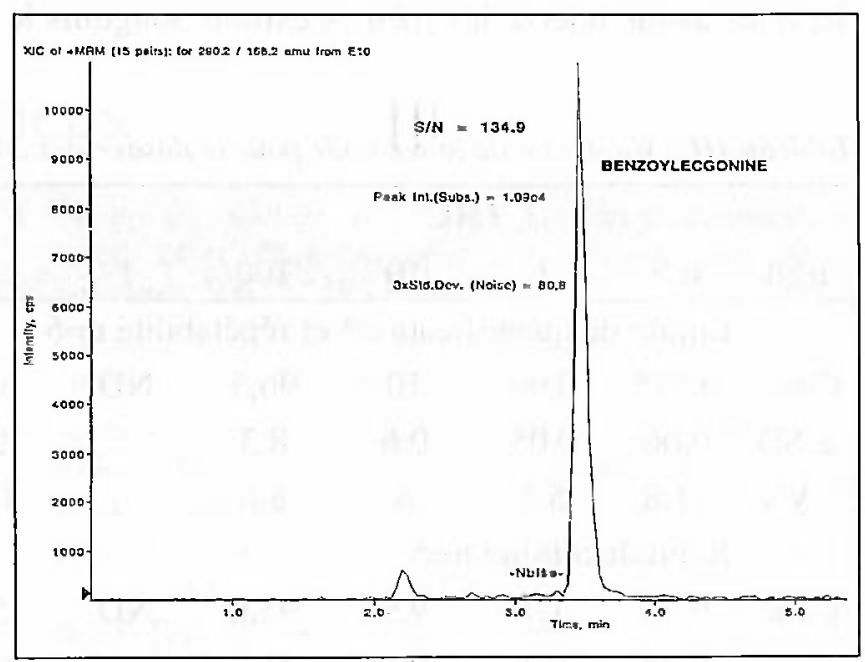

Figure 2 : Chromatogramme d'un extrait sanguin surchargé par $10 \mu \mathrm{g} / \mathrm{l}$ de benzoylecgonine. 


\section{Les cannabinoïdes}

La méthode d'ionisation utilisée est l'APCI qui améliore l'intensité du signal d'un facteur 10 environ par rapport à l'ESI pour le THC. Les validations de méthode pour les cannabinoïdes ont été faites avec ce mode d'ionisation.

Les résultats de validation de la méthode sont présentés sur le tableau III. Dans le sang total, la méthode est linéaire jusqu'à $100 \mu \mathrm{g} / \mathrm{l}$, des valeurs supérieures n'ont pas été testées. L'analyse de la justesse montre des taux de récupération compris entre $85 \%$ et $115 \%$. Les coefficients de variation mesurés lors de l'étude de répétabilité et de reproductibilité sont inférieurs ou égaux à $15 \%$.

La limite de quantification, définie comme étant la concentration la plus basse pour laquelle les critères d'acceptation de fidélité (coefficient de variation inférieur à $20 \%$ ) et de justesse (pourcentage de récupération compris entre $80 \%$ et $120 \%$ ) sont respectés, est estimée à $0,5 \mu \mathrm{g} / 1$ pour le $\mathrm{THC}$, à $1 \mu \mathrm{g} / \mathrm{l}$ pour le $\mathrm{THC}-\mathrm{COOH}$ et seulement à $5 \mu \mathrm{g} / \mathrm{l}$ pour le $\mathrm{OH}-\mathrm{THC}$, ce composé n'est probablement pas suffisamment ionisé dans les conditions analytiques utilisées ; cependant les limites de quantification des 2 autres cannabinoïdes sont suffisantes pour affirmer s'il y a eu usage de cannabis.

Nous avons eu la possibilité de tester une source récemment commercialisée : la source à photoionisation ou source APPI. Cette source a un mode de fonctionnement et une géométrie très proches de celui de la source APCI. Les différences essentielles résident dans le fait de vaporiser un dopant (en général le toluène) dans la source. Les molécules de dopant sont ionisées par les radiations UV d'une lampe au Xénon et produisent des photoions. Ceux-ci entraînent des réactions ions/molécules en cascade conduisant à la formation d'ions par transfert de proton. Nous ne présenterons ici que quelques résultats préliminaires. Dans le but de tester une amélioration éventuelle de la méthode, nous avons injecté les mêmes extraits sanguins le même jour en utilisant les sources APCI et APPI. Un exemple de chromatogramme est présenté sur la figure 3. Les extraits faits sur des échantillons sanguins recueillis lors d'un contrôle de la sécurité routière ont été mesurés à $1,5 \mu \mathrm{g} / \mathrm{l}$ pour le THC. Les résultats concernant le THC montrent une augmentation nette du signal et du rapport signal/bruit d'environ un facteur 3 pour ce composé. Nous avons constaté également une amélioration de la détection pour le $\mathrm{OH}-\mathrm{THC}$, mais pas pour le THC-COOH. Les coefficients de variation mesurés lors de l'étude de répétabilité en mode APPI sont égaux à $8,5 \%$ pour le THC à une concentration de $0,5 \mu \mathrm{g} / \mathrm{l}$ et respectivement égaux à $8 \%$ et $13 \%$ pour le THC-COOH et le $\mathrm{OH}-\mathrm{THC}$ à une concentration de $1 \mu \mathrm{g} / \mathrm{l}$. Ces résultats indiquent qu' avec une source APPI on obtient une meilleure ionisation pour les composés les moins polaires.

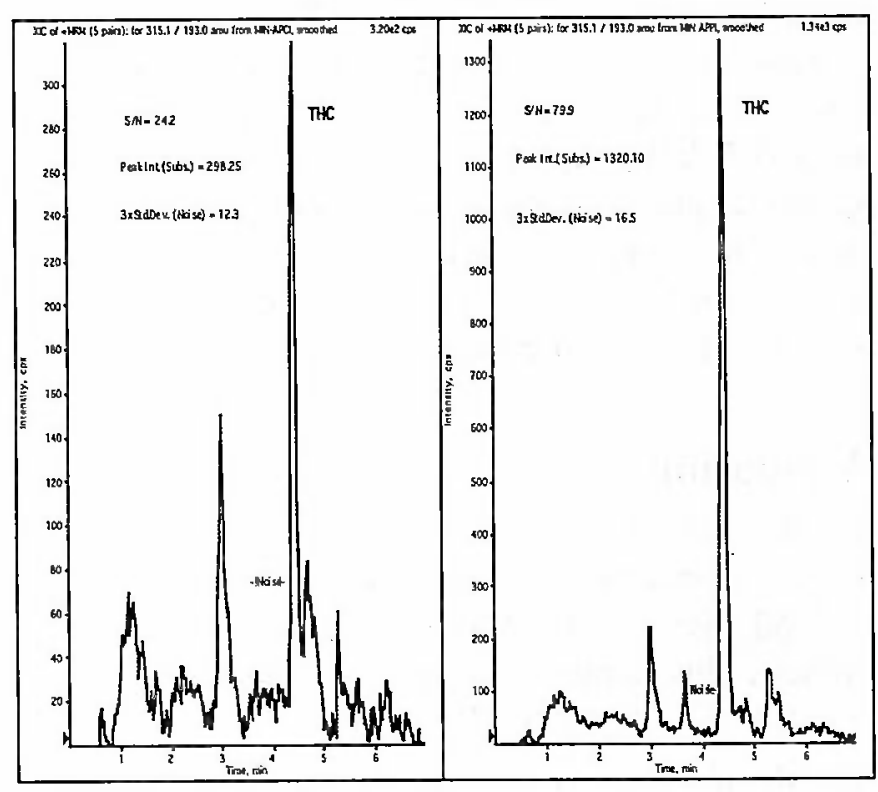

Figure 3 : Chromatogramme d'un extrait sanguin d'un conducteur impliqué dans un accident de circulation routière; THC mesuré à $1,5 \mu \mathrm{g} / \mathrm{l}$ à gauche ionisation en mode $A P C I$, à droite ionisation en mode APPI.

Tableau III : Validation de la méthode pour le dosage des cannabinö̈des dans le sang.

\begin{tabular}{|c|c|c|c|c|c|c|c|c|c|c|c|c|}
\hline \multirow[b]{2}{*}{$\mu \mathrm{g} / \mathrm{I}$} & \multicolumn{4}{|c|}{ THC } & \multicolumn{4}{|c|}{ OH-THC } & \multicolumn{4}{|c|}{ THC-COOH } \\
\hline & 0,5 & 1 & 10 & 100 & 1 & 5 & 10 & 100 & 0.5 & 1 & 10 & 100 \\
\hline \multicolumn{13}{|c|}{ Limite de quantification* et répétabilité $n=6$} \\
\hline Conc. & $0,51 *$ & 0,90 & 10 & 96,5 & ND & $5,2 *$ & 10,8 & 107,7 & 0,62 & $1,0^{*}$ & 10,2 & 93,0 \\
\hline$\pm \mathrm{SD}$ & 0,06 & 0,05 & 0,6 & 8,3 & & 0,9 & 0,7 & 11,3 & 0,16 & 0,09 & 0,6 & 6 \\
\hline CV\% & 11,8 & 5,5 & 6 & 8,6 & & 17,3 & 6,5 & 10,5 & 25,8 & 5,7 & 5,9 & 6,5 \\
\hline \multicolumn{13}{|c|}{ Reproductibilité $n=5$} \\
\hline Conc. & 0,53 & 1,1 & 9,9 & 95,6 & ND & 5,3 & 10,5 & 102 & & 1,1 & 9,9 & 92,1 \\
\hline$\pm \mathrm{SD}$ & 0,08 & 0,1 & 0,8 & 11,0 & & 1,05 & 1 & 13 & & 0,16 & 0,7 & 8,2 \\
\hline $\mathrm{CV} \%$ & 15,1 & 11,3 & 8 & 11,5 & & 19,8 & 9,5 & 12,7 & & 14,5 & 7 & 8,9 \\
\hline
\end{tabular}


Les méthodes que nous avons décrites, semblent adaptées à l'analyse des stupéfiants recherchés dans le cadre de la sécurité routière. Sur le tableau IV sont reportés les résultats que nous avons obtenus pour les contrôles de qualité SFTA dans le sang; à l'exception de 2 valeurs, les résultats rendus se trouvent dans l'intervalle de confiance.

\section{Conclusion}

Le spectromètre de masse en tandem couplé à un chromatographe en phase liquide est un outil particulièrement bien adapté pour l'analyse des stupéfiants demandés dans le cadre de la circulation routière. Il est néces- saire de rechercher le mode d'ionisation le mieux adapté à l'analyte à mesurer : ionisation electrospray (ESI), chimique (APCI) ou photoionisation (APPI). Les méthodes sont rapides, ne nécessitent pas de dérivation préalable à l'injection, ce qui limite les problèmes liés à l'encrassement et au nettoyage de la source. Plusieurs classes de stupéfiants peuvent être recherchées et mesurées dans une même analyse chromatographique. La recherche et le dosage des amphétamines sont également possibles par LC-MS/MS et ionisation en mode electrospray. La méthode est en cours de validation au laboratoire ce qui permettra d'obtenir opiacés, cocaïne et ses métabolites ainsi que les amphétamines dans une seule analyse LC-MS/MS après ionisation en mode electrospray.

Tableau IV : Résultats des contrôles qualité des stupéfiants dans le sang (italique : intervalle des contrôles) (*: valeur en dehors des limites).

\begin{tabular}{|c|c|c|c|c|c|}
\hline $\begin{array}{c}\text { année } \\
\text { Composés }(\mu g / 1)\end{array}$ & 2000 & 2001 & 2002 & 2003 & 2004 \\
\hline Morphine & & $\begin{array}{c}\mathbf{8 5 *} \\
37-75\end{array}$ & $\begin{array}{c}53 \\
30-90\end{array}$ & $\begin{array}{l}14,3 \\
8-20\end{array}$ & $\begin{array}{c}36 \\
18-45\end{array}$ \\
\hline Codéine & & $\begin{array}{c}175 \\
94-191\end{array}$ & $\begin{array}{c}79 \\
64-109\end{array}$ & & $\begin{array}{c}14 \\
6-28\end{array}$ \\
\hline éthylmorphine & $\begin{array}{c}75 \\
66-110\end{array}$ & & & & \\
\hline Benzoylecgonine & négatif & $\begin{array}{c}91 \\
54-120\end{array}$ & $\begin{array}{c}\mathbf{8 3} \\
61-103\end{array}$ & $\begin{array}{c}\mathbf{8 , 6 *} \\
10-35\end{array}$ & $\begin{array}{c}49 \\
36-64\end{array}$ \\
\hline THC & $\begin{array}{c}\mathbf{5 , 7} \\
4,3-7,0\end{array}$ & $\begin{array}{c}15 \\
7-23\end{array}$ & $\begin{array}{c}4 \\
2,6-6,8\end{array}$ & $\begin{array}{c}<1 \\
0,2-1\end{array}$ & $\begin{array}{c}41 \\
24-67\end{array}$ \\
\hline THC-COOH & & $\begin{array}{c}25 \\
17-32\end{array}$ & $\begin{array}{c}\mathbf{2 1} \\
13-28,2\end{array}$ & $\begin{array}{c}7,4 \\
3,4-8,8\end{array}$ & \\
\hline
\end{tabular}

\section{Références}

1. Loi $\mathrm{n}^{\circ}$ 99-505 du 18 juin 1999 portant diverses mesures relatives à la sécurité routière et aux infractions sur les agents des exploitants de réseau de transport public de voyageurs.

2. Loi relative à la conduite sous l'influence de substances ou plantes classées comme stupéfiants adoptée en seconde lecture par l'Assemblée Nationale le 23 janvier 2003, loi 2003-87 du 3 février 2003, publiée au JO du 4 février 2003 et modifiée par loi $n^{\circ} 2003-495$ du 12 juin 2003 renforçant la lutte contre la violence routière.

3. Cone E. J., Huestis M. A. Relating blood concentrations of tetrahydrocannabinol and metabolites to pharmacologic effects and time of marijuana usage. Therapeut. Drug Monitor. 1993 ; 15 : 527-32.
4. Skopp G., Richter B., Potsch L. Serum cannabinoid levels 24 to 48 hours after cannabis smoking. Arch. Kriminol. $2003 ; 212: 83-95$.

5. Cirimele V., Kintz P., Mangin P. Testing human hair for cannabis. Fore. Sci. Int. $1995 ; 70: 175-82$.

6. Kintz P., Cirimele V., Pépin G., Marquet P., Deveaux M., Mura P. Identification et dosage des cannabinoïdes dans le.sang total. Toxicorama $1996 ; 8: 29-33$.

7. Giroud C., Menetrey A., Augsburger M., Buclin T., Sanchez-Mazas P. delta'-THC, 11-OH-delta ${ }^{9}-\mathrm{THC}$ and delta ${ }^{9}$-THC-COOH plasma or serum to whole blood concentrations distribution ratios in blood samples taken from living and dead people. Fore. Sci. Int. 2001 ; 123 : 159-64. 
8. Moeller M. R., Doerr G., Warth S. Simultaneous quantitation of delta-9-tetrahydrocannabinol (THC) and 11nor-9-carboxy-delta-9-tetrahydrocannabinol (THC$\mathrm{COOH}$ ) in serum by GC-MS using deuterated internal standards and its application to a smoking study and forensic cases. J. Forensic Sci. 1992 ; 37 : 969-83.

9. Menetrey A., Augsburger M., Rothuizen L. E., Pin M. A., Appenzeller M., Favrat B., Buclin T., Mangin P., Giroud C. Profils cinétiques du D9-tetrahydrocannabinol, du 11hydroxy-D9-tétrahydrocannabinol et de l'acide 11-norD9-tétrahydrocannabinol-9-carboxylique chez le sujet volontaire sain après administration orale de décoctions de cannabis ou de dronabinol. Ann. Toxicol. Anal. $2004 ; 16: 231-43$.

10. Meadway C., George S., Braithwaite R. A rapid GC-MS method for the determination of dihydrocodeine, codeine, norcodeine, morphine, normorphine and 6-MAM in urine. Forensic Sci. Int. 2002 ; 127, Issues 1-2 : 136-41.

11. Pépin G., Dubourvieux N. and Chèze M. Opiacés et opioïdes : usages, effets, dépistage, dosage. Rev. Fr. Labor., 2000 ; Issue 322 : 41-5.

12. Romolo F. S., Rotolo M. C., Palmi I, Pacifici R. and Lopez A. Optimized conditions for simultaneous determination of opiates, cocaine and benzoylecgonine in hair samples by GC-MS. Forensic Sci. Int., 2003 ; 138, Issues $1-3,17: 17-26$.

13. Bogusz M. J., Maier R. D., Krüger K. D., Kohls U. Determination of common drugs of abuse in body fluids using one isolation procedure and liquid-chromatography atmospheric-pressure chemical ionisation mass spectrometry. J. Anal. Toxicol. 1998 ; 22 : 549-58.

14. Cailleux A., Le Bouil A., Turcant A., Allain P. Identification et dosage des opiacés dans les milieux biologiques par chromatographie en phase liquide couplée à la spectrométrie de masse en tandem. Toxicorama 1999 ; $11: 90-4$.

15. Cailleux A., Le Bouil A., Auger B., Bonsergent G., Turcant A., Allain P. Determination of opiates and cocaine and its metabolites in biological fluids by high-performance liquid chromatography with electrospray tandem mass spectrometry, J. Anal. Toxicol. 1999 ; 23 : 620-5.

16.Canezin J., Cailleux A., Turcant A., Le Bouil A., Harry P., Allain P., Determination of LSD and metabolites in human biological fluids by high-performance liquid chromatography with electrospray tandem mass spectrometry. J. Chromatogr. B $2001 ; 765: 15-27$.

17. Weinmann W., Vogt S., Goerke R., Müller C., Bromberger A. Simultaneous determination of THC$\mathrm{COOH}$ and $\mathrm{THC}-\mathrm{COOH}$ glucuronide in urine samples by LC/MS/MS. Forensic Sci. Int. $2000 ; 113: 381-7$.

18. Weinmann W., Goerner M., Vogt S., Goerke R., Pollak S. Fast confirmation of 11-nor-9-carboxy-delta"-tetrahydrocannabinol (THC-COOH) in urine samples by LC/MS/MS using negative atmospheric-pressure chemical ionisation (APCI). Forensic Sci. Int. $2001 ; 121$ : 103-7.
19. Marquet P., Saint-Marcoux F., Gamble T. N., Leblanc J. C. Y. Comparison of a preliminary procedure for the general unknow screening of drugs and toxic compounds using a quadrupole linear ion trap mass spectrometer with a liquid chromatography-mass spectrometry reference technique. J. Chrom. B 2003 ; 789 : 9-18.

20. Maralikova B., Weinmann W. Simultaneous determination of Delta9-tetrahydrocannabinol, 11-hydroxy-Delta9tetrahydrocannabinol and 11-nor-9-carboxy- Delta9tetrahydrocannabinol in human plasma by high-performance liquid chromatography/tandem mass spectrometry. J. Mass Spectrom. 2004 ; 39(5) : 526-31.

21. Valitevi S., Stinchomb AL. Liquid chromatographicmass spectrometric quantitation of Delta9-tetrahydrocannabinol and two metabolites in pharmacokinetic study plasma samples. J. Chrom. B Analyt. Technol. Biomed. Life Sci. 2004 ; 803(2) : 243-8.

22. Gustafson R. A., Moolchan E. T., Barnes A., Levine B., Huestis M. A. Method for the simultaneous determination of Delta 9-tetrahydrocannabinol (THC), 11hydroxy-THC and 11-nor-9-carboxy-THC in human plasma using solid phase axtraction and gas chromatography-mass spectrometry with positive chemical ionization. J. Chrom. B Analyt. Technol. Biomed. Life Sci. $2003: 798(1): 145-54$.

23. Maralikova B., Weinmann W. Confirmatory analysis for drugs of abuse in plasma and urine by high-performance liquid chromatography-tandem mass spectrometry with respect to criteria for compound identification . J. Chrom. B, $2004 ; 811$, Issue 1 : 21-30.

24. Pihlainen K., Sippola E. I., Kostiainen R. Rapid identification and quantitation of compounds with forensic interest using fast liquid chromatography-ion trap mass spectrometry and library searching J. Chrom. A, 2003 ; 994 : 93-102.

25. Uhl M. Tandem mass spectrometry : a helpful tool in hair analysis for the forensic expert. Forensic Sci. Int, 2000 ; 107 : 169-79.

26. Kronstrand R., Nyström I., Strandberg J., Druid H. Screening for drugs of abuse in hair with ionspray LCMS-MS. Fore. Sci. Int. 2004 ; 145 : 183-90.

27. Maurer $\mathrm{H}$. Liquid chromatography-mass spectrometry in forensic and clinical toxicology. J. Chrom. B, 1998 ; $713: 3-25$.

28. Thieme D., Sachs H. Improved screening capabilities in forensic toxicology by application of liquid chromatography-tandem mass spectrometry. Anal Chim. Act., 2003 ; 492 : 171-86. 